\title{
ALTERNATIVAS PARA SUPLIR LA CARENCIA DE FÓSFORO EN FRESA Y DISMINUIR LA CONTAMINACIÓN AMBIENTAL EN PAMPLONA NORTE DE SANTANDER
}

\section{ALTERNATIVES TO SUPPLY THE LACK OF PHOSPHORUS IN STRAWBERRIES AND REDUCE ENVIRONMENTAL CONTAMINATION IN PAMPLONA NORTE DE SANTANDER}

\author{
Jorge Gonzalo Mahecha Molina, Leónides Castellanos González, Néstor \\ Céspedes Novo
}

\section{Entidad}

Facultad de Ciencias Agrarias, Universidad de Pamplona, Carretera a Bucaramanga Km 1, Pamplona, Norte de Santander, Colombia. Jefe de Proyecto, Asociación de Productores Agropecuarios de Pamplona, Norte de Santander, Colombia. E-mail: jorgemahecha@hotmail.com, lclcastell@gmail.com, nestorcespedeshse@gmail.com

\section{Resumen}

El presente trabajo tuvo como objetivo evaluar seis bioproductos locales como alternativas orgánicas para suplir la carencia de fósforo en fresa, en Pamplona Norte de Santander. Se desarrolló un ensayo de campo con ocho tratamientos, seis biopreparados elaborados por ASPAGRO, un fertilizante químico convencional (Folyfos) y un testigo. Caldo Rizósfera y M6 mostraron mayor disminución en la incidencia y severidad de la deficiencia de fósforo, comparados con el testigo y los demás tratamientos. Los tratamientos Caldo Rizósfera y M6 se destacaron con relación a la mayor concentración de fosforo foliar, con una correlación negativa y significativa con la incidencia y la severidad de los síntomas de la deficiencia de fósforo.

\section{Palabras clave}

Nutrición, carencia fosfórica, biopreparados, microrganismos eficientes, medio ambiente

\begin{abstract}
El presente trabajo tuvo como objetivo evaluar seis bioproductos locales como alternativas orgánicas para suplir la carencia de fósforo en fresa, en Pamplona Norte de Santander. Se desarrolló un ensayo de campo con ocho tratamientos, seis biopreparados elaborados por ASPAGRO, un fertilizante químico convencional (Folyfos) y un testigo. Caldo Rizósfera y M6 mostraron mayor disminución en la incidencia y severidad de la deficiencia de fósforo, comparados con el testigo y los demás tratamientos. Los tratamientos Caldo Rizósfera y M6 se destacaron con relación a la mayor concentración de fosforo foliar, con una correlación negativa y significativa con la incidencia y la severidad de los síntomas de la deficiencia de fósforo.
\end{abstract}

Key words:

nutrición, carencia fosfórica, biopreparados, microrganismos eficientes, enviroment. 


\section{INTRODUCCIÓN}

El cultivo de fresa tiene una gran importancia socioeconómica en clima frio, pero para lograr rendimientos óptimos y rentables se requiere de investigación para un mejor manejo del cultivo, sobre todo para el caso de macronutrientes y micronutrientes, como son los elementos fundamentales garantizan la producción final, porque la planta requiere para beneficios fisiológicos, productivos, de resistencia a plagas y enfermedades, por las indagaciones de este tipo en literatura son escasas (Cano, 2013; Villamizar \& Fernández, 2015).

La fruta de fresa tiene un alto nivel mundial de consumo ya sea directo o en elaboración industrial de postres, bebidas. Con muchos métodos de conservación deshidratada, congelada, hasta en conservas. Por el gran consumo se ha llegado a trabajar por un mejoramiento genético y con investigaciones para el desarrollo de mejor producción en la parte de manejo agronómico como lo es la fertilización, para tener cultivos resistentes a los diferentes cambios repentinos que se presenten como son heladas, ataque de plagas y enfermedades (Marquez, 2001).

Colombia está entre los países de mayor exportación de fresa por lo que debe buscar alternativas de cultivos orgánicos para tener mejores mercados internacionales, brindarle al consumidor un fruto libre de pesticidas (Casierra \& Poveda, 2005). Deben realizarse investigaciones que permitan reducir el uso de productos químicos como fertilizantes, insecticidas y fungicidas, porque al ser aplicados, además de llevar trazas en las cosechas para el consumidor, también favorecen la erosión del suelo y perdidas de las características físicas, químicas y biológicas del mismo, remplazándolos por biopreparados orgánicos con el fin de bríndale al consumidor de frutas de fresa, un producto sano, libre de enfermedades y de residuos químicos (Casierra, 2011; Rivera et al, 2017).

El fosforo es un elemento esencial para el desarrollo de la planta de fresa, no se logran los rendimientos potenciales si no tiene la cantidad de fósforo suficiente, ya que este es vital en el almacenamiento de energía y la regulación del metabolismo. Aumentando la resistencia sistemática al ataque de plagas, las plantas de fresa con fertilizadas con fósfitos modifica el sabor de la fruta (Estrada \& Iris, 2011).

Los Microorganismos Eficientes (ME) son una combinación de microorganismos beneficiosos de cuatro grupos principales: bacterias fototróficas, levaduras, bacterias productoras de ácido láctico y hongos de fermentación. Estos microorganismos contienen microorganismos fijadores de $\mathrm{N}$ atmosféricos y solubilizadores de fósforo entre otros (BID, 2009), mientras que los Microorganismos de Montaña (MM) se podrían catalogar como un tipo de ME, o sea, un consorcio de microbianos ya que su composición y las posibles relaciones que generan son múltiples, se indica que contienen bacterias fotosintéticas, bacterias productoras de ácido láctico, actinomicetes, hongos filamentosos y levaduras que también ayudan a la solubilización de P (Castro et al., 2015).

Moreno (2011) realizó un estudio sobre la aplicación de dos fosfitos artesanales en el cultivo de fresa (Fragaria vesca) en Ecuador con el fin de reducir la incidencia de la mancha foliar (Cercospora sp) donde se usó como sustrato 1; una mezcla de cascarilla de arroz $30 \mathrm{~kg}$, roca fosfórica 1,5 $\mathrm{kg}$ y harina de hueso $1,5 \mathrm{~kg}$. Como sustrato 2; se usó $30 \mathrm{~kg}$ de cascarilla de arroz, $1,5 \mathrm{~kg}$ de roca fosfórica y $1,5 \mathrm{~kg}$ de carbonato de calcio y se aplicaron dos tratamientos con dosis uno de $125 \mathrm{~g} / 200 \mathrm{~L}$, dosis dos de $250 \mathrm{~g} / 200 \mathrm{~L}$, dosis tres 375 g/200 L. Después de 30 días de estar aplicando los productos se redujo la enfermedad siendo mejor el fosfito a la 
dosis de $375 \mathrm{~g} / 200 \mathrm{~L}$, que redujo la severidad de los síntomas en un $65 \%$.

Lara \& Negrete (2015) evaluaron la capacidad solubilizadora de fosfatos de consorcios formados por bacterias nativas de los géneros Burkholderia cepacia, Pseudomonas sp, Pseudomonas luteola y Pantoea sp, en Córdoba, Colombia, con resultados satisfactorios. Medina, pinzón \& Cely (2016) realizaron un estudio del efecto de sustratos orgánicos en plantas de fresa (Fragaria sp.) cv 'Albion' bajo condiciones de campo en Tunja, Boyaca. Un tratamiento, mostró diferencias significativas en la mayoría de los parámetros evaluados, esto lo convierte en una alternativa para la producción de fresa cv 'Albión' bajo las condiciones de estudio.

Gualdron y Maldonado (2017) enfatizan sobre la necesidad de buscar alternativas menos contaminantes con el medio ambiente en las zonas agricolas de Pamplona, Norte de Santander, mientras que Mahecha, Castellanos, y Céspedes (2019), han informado que los problemas de carecía de fósforo en fresa están bastante extendidos en las diferentes variedades y veredas de Pamplona, donde se aplican altas cantidades de fertilizantes con este elemento que además encarecen la producción (Ortega, 2016; Meneses et al, 2016).

El presente trabajo se realizó con el objetivo de evaluar alternativas a los problemas que se presentan en el cultivo de fresa (Fragaria vesca L.) por deficiencia de fósforo, usando seis productos biopreparados de la Asociación de Productores Agropecuarios de Pamplona (ASPAGRO), los cuales contienen microorganismos eficientes, microorganismos de montaña y otros obtenidos en la localidad que pudieran solubilizar el fósforo en el suelo.

\section{METODOLOGIA}

Se desarrolló un experimento de campo en el periodo de junio a octubre de 2017 en un lote de un cultivo de fresa con una edad de ocho meses de plantada en un área de una superficie de $1498,4 \mathrm{~m}^{2}$ de la finca Camachos, vereda Cariongo del municipio de Pamplona.

En esa área se había realizado un análisis agroquímico del suelo arrojando las siguientes características: $\mathrm{pH}$ de 5.6, carbono orgánico o contenido de materia orgánica $6,82 \%$ y fósforo $2,40 \mathrm{mg} / \mathrm{kg}$. El contenido de materia orgánico se catalogaba medio, el contenido de $\mathrm{P}$ bajo y el pH bajo.

Inicialmente se realizó un saneamiento de todas las plantas, hojas, flores y frutos necrosados en las parcelas donde se aplicaron los productos biológicos. Además, se realizó una aplicación materia orgánica con una dosis de $400 \mathrm{~kg} / \mathrm{ha}$.

Los tratamientos fueron los siguientes (Tabla 1)

Tabla 1. Tratamientos y diseño experimental

1. Testigo sin tratamiento con saneamiento.

2. Biopreparado 1: $P_{1}$

3. Biopreparado 2: $\mathrm{P}_{2}$

4. Biopreparado 3: MM

5. Biopreparado 4: Caldo rizosfera

6. Biopreparado 5: B. meconio

7. Biopreparado 6: $M_{6}$

8. Aplicación foliar de $\mathrm{P}_{2} \mathrm{O}_{5}$

Los tratamientos se dispusieron en un diseño experimental de bloques al azar en arreglo $8 \times 4$ (ocho tratamientos y cuatro replicas). Los tratamientos consistieron en seis los seis biopreparados ASPAGRO, uno estándar de producción con la aplicación de fertilizante químico foliar $\left(\mathrm{P}_{2} \mathrm{O}_{5}\right)$ y un testigo con saneamiento como se relacionó anteriormente. Cada tratamiento fue replicado cuatro veces en parcelas de tres eras y 13, $86 \mathrm{~m}$ de largo por lo que las parcelas 0 unidades experimentales contaron con 41, $65 \mathrm{~m}^{2}$. 
A continuación, se relacionan los ingredientes de cada biopreparado Suministrados por ASPAGRO y tipo de fermentación durante el proceso de producción.

Tabla 2. Descripción de cada biopreparado

\begin{tabular}{|c|c|c|}
\hline $\begin{array}{l}\text { Biopreparados } \\
\text { comerciales }\end{array}$ & Ingredientes & Uso propuesto \\
\hline P1 & $\begin{array}{l}\text { Vinagre, roca } \\
\text { fosfórica y melaza }\end{array}$ & $\begin{array}{l}\text { Biofertilizante } \\
\text { con B.S.F. } \\
8,34 \times 10^{5} \times \\
\text { UCF } / \mathrm{mL}\end{array}$ \\
\hline $\mathrm{P} 2$ & $\begin{array}{l}\text { ME y melaza, } \\
\text { ceniza } \\
\text { fosfórica }\end{array}$ & $\begin{array}{l}\text { Biofertilizante } \\
\text { con B.S.F. y } \\
\text { antagonista } \\
3.2 \quad x \\
\text { UCF/mL }\end{array}$ \\
\hline MM & $\begin{array}{l}\text { ME, melaza y } \\
\text { salvado de arroz }\end{array}$ & $\begin{array}{l}\text { Biofertilizante } \\
\text { con B.S.F. y } \\
\text { antagonista } \\
1,72 \times 10^{6} \text { UFC } / \mathrm{mL}\end{array}$ \\
\hline $\begin{array}{l}\text { Caldo } \\
\text { rizosfera }\end{array}$ & $\begin{array}{l}\text { Raíces de plantas } \\
\text { de la granja, yogurt, } \\
\text { melaza, agua } \\
\text { oxigenada y harina } \\
\text { de frijol }\end{array}$ & $\begin{array}{l}\text { Biofertilizante } \\
\text { con B.S.F. y } \\
\text { antagonista } \\
1,24 \times 10^{6} \text { UFC } / \mathrm{mL}\end{array}$ \\
\hline B. meconio & $\begin{array}{l}\text { Meconio de ternero, } \\
\text { agua, melaza y } \\
\text { ahuyama cocida. }\end{array}$ & $\begin{array}{l}\text { Biofertilizante } \\
\text { con B.S.F. y } \\
\text { antagonista } \\
1,3 \times 10^{6} \text { UFC/mL }\end{array}$ \\
\hline M6 & $\begin{array}{l}\text { ME, vinagre, etanol, } \\
\text { plantas aromáticas, } \\
\text { jengibre, ajo, } \\
\text { cebolla, pimienta y } \\
\text { ají. }\end{array}$ & $\begin{array}{l}\text { Biofertilizante } \\
\text { con B.S.F. y } \\
\text { antagonista } \\
9,13 \times 10^{5} \text { UFC } / \mathrm{mL}\end{array}$ \\
\hline
\end{tabular}

Nota: características de los biopreparados usados en la evaluación (Fuente: Castellanos, et al., 2017)

Los productos de biopreparados se aplicaron semanalmente sobre las plantas y el suelo a las dosis recomendadas por ASPAGRO de una solución de $400 \mathrm{~L} / \mathrm{ha}$, con una bomba de espalda manual con boquilla de abanico, como los tratamientos por estudiar de encuentran cubiertos por plástico se realizará una aplicación a 10 a 15 centímetros de altura de la planta.

Las dosis los biopreparados fue del $5 \%$ (1L/20L), con excepción del biopreparado B. meconio que se aplicó al $1 \% 200$ $\mathrm{mL} / 20 \mathrm{~L}$.

Se evaluaron 20 plantas tomadas al azar de los cuatro surcos centrales de cada parcela por tratamiento se determinaron las siguientes variables:

Porcentaje de Incidencia y de Severidad de los síntomas de deficiencia de fósforo cada cuatro semanas.

Para la determinación de la Incidencia y la Severidad de los síntomas de deficiencia de $P$ se empleó la escala de 6 grados referida anteriormente:

Escala

$\mathbf{0}=$ planta sin síntomas de deficiencia.

1= la planta presenta un síntoma de deficiencia entre un $5 \%$ del área foliar.

2= la planta presenta un síntoma de deficiencia entre un $6-25 \%$ del área foliar.

3= la planta presenta un síntoma de deficiencia entre un 26 - $50 \%$ del área foliar.

4= la planta presenta un síntoma de deficiencia entre un 51 - $75 \%$ del área foliar.

5= la planta presenta un síntoma de deficiencia mayor de un $76 \%$ del área foliar.

Las fórmulas que se utilizarán serán:

Con la información obtenida en los muestreos se determinó el Porcentaje de Incidencia por la siguiente formula:

$\%$ Inc. $=\frac{n(\text { plantas con deficiencias })}{N \text { (plantas evaluadas })} X 100$

Así como la Severidad del síntoma de carencia de fósforo. Este último indicador es un indicador de porcentaje de tejido afectado por la deficiencia del elemento Se calculó por la fórmula de Towsend y Heuberger.

$\% S=\frac{\sum a * b}{K N} X 100$

Donde:

$\mathrm{S}=$ Severidad

$\mathrm{a}=$ Grado de la escala

$\mathrm{b}=$ Número de plantas con un grado a de la escala

$\mathrm{K}=$ Grado máximo de la escala

$\mathrm{N}=$ Número total de plantas muestreadas 
A los 150 días se realizó un análisis del contenido de $P$ foliar tomando una muestra representativa de cada parcela la cual fue llevada al Laboratorio de Química de la Unipamplona.

Para determinar el contenido de fósforo del área foliar se procedió a recolectar 60 hojas en 60 plantas (la tercera hoja bajando) en de cada replica de los ocho tratamientos, luego se llevó al horno por una semana, y se procedió hacer un molido o macerado de cada muestra foliar. Posteriormente se pesaron 2,5 g de muestra macerada y se llevaron a un crisol y se realizó un previo quemado con la ayuda de un mechero, luego se llevaron las muestras a la mufla a $350^{\circ} \mathrm{C}$.

Cuando las muestras estaban de nuevo a temperatura ambiente se agregaron $25 \mathrm{ml}$ de ácido clorhídrico a cada crisol, se procedió a evaporar en una plancha de calentamiento, hasta que quedaran $10 \mathrm{ml}$ de la solución, luego se filtró cada muestra con la ayuda de papel filtro en tubos de ensayo, agregándole a cada muestra $3 \mathrm{ml}$ de solución A para identificación de concentración de área foliar de fósforo y 5 $\mathrm{ml}$ de solución B. Se agitó cada muestra hasta que tomó un color azul.

Finalmente, el resultado de cada muestra se llevó al fotocolorímetro de marca $\mathrm{HACH}$ (DR 3800) para el caso de fósforo de espectrofotómetro 489 fosfato, para obtener la lectura del contenido de $P$ en $\mathrm{mg} / \mathrm{L}$, para luego hacer los cálculos para estimar las partes por millón (ppm) que tenía cada muestra por medio de la siguiente formula.

ppm $(\mathrm{mg} / \mathrm{kg})=\frac{\text { peso de la muestra }}{\mathrm{mg} / \mathrm{L} \text { de cada muestra }} * 1000$

Se realizó un análisis de varianza con todas las variables en los diferentes muestreos (excepto las económicas) una vez comprobado el supuesto de normalidad por la prueba de Kolmodorov Smirnov. Se compararon las medias por la prueba de Tukey, con un 5\% probabilidad de error.

Se realizó un análisis correlación y regresión entre la concentración foliar de fósforo al final del experimento por parcela como variante independiente, y la Incidencia y la Severidad de los síntomas en la última evaluación del experimento como variables independientes. Para estos análisis estadísticos se empleó el paquete estadístico SPSS o ASSISTAT.

\section{ANALISIS $Y$ DISCUSIÓN DE RESULTADOS}

Los datos tomados a la cuarta semana de haber iniciado la aplicación de los productos se detectaron con menor incidencia de los síntomas de carencia de fósforo los tratamientos de los biopreparados $P_{1}, M_{6}, M M, B$. meconio, $P_{2}$, el tratamiento con $\mathrm{P}_{2} \mathrm{O}_{5}$, y el testigo sin tratamiento que no difirieron estadísticamente entre sí. El tratamiento de mayor nivel de incidencia fue Caldo Rizósfera, aunque $\mathrm{B}$. meconio y $\mathrm{P}_{2}$ no se diferenciaron de este. En la variable severidad de la carencia de $P$ en ese momento no se observó diferencia entre los tratamientos y el testigo (Tabla 3).

Tabla 3. Incidencia y severidad de los síntomas de deficiencias de $\mathrm{P}$ en los diferentes tratamientos en el primer muestreo de la cuarta semana (17 de junio del 2017)

\begin{tabular}{|c|c|c|c|c|c|}
\hline \multirow{2}{*}{\multicolumn{2}{|c|}{ Tratamientos }} & \multirow{2}{*}{\multicolumn{2}{|c|}{$\begin{array}{l}\text { Incidencia } \\
(\%)\end{array}$}} & \multicolumn{2}{|c|}{ Severidad } \\
\hline & & & & (\%) & \\
\hline 1 & Testigo & 70,0 & $b$ & 27,5 & $a$ \\
\hline 2 & $\mathbf{P}_{1}$ & 72,5 & $b$ & 30,5 & $\mathrm{a}$ \\
\hline 3 & $\mathbf{P}_{2}$ & 82,5 & $a b$ & 29,5 & $\mathrm{a}$ \\
\hline 4 & MM & 70,0 & $b$ & 23,0 & $\mathrm{a}$ \\
\hline 5 & C. Rizósfera & 87,5 & a & 29,5 & $\mathrm{a}$ \\
\hline 6 & B. meconio & 75,0 & $a b$ & 30,5 & $\mathrm{a}$ \\
\hline 7 & M6 & 67,5 & $b$ & 22,5 & $\mathrm{a}$ \\
\hline & $\mathrm{P}_{2} \mathrm{O}_{5}$ & 72,5 & $b$ & 24,0 & $\mathrm{a}$ \\
\hline & V. (\%) & 1,00 & & 1,53 & \\
\hline & ror Típico & 0,07 & & 0,07 & \\
\hline
\end{tabular}


Datos tomados en bloques al azar en cada uno de los tratamientos (Fuente: Propia)

En el muestreo de la semana 8 la incidencia de los síntomas de carencia fósforo fue menor en Los tratamientos Caldo Rizósfera y M6 se diferenciaron del testigo, el biopreparado P1 y del tratamiento con $\mathrm{P}_{2} \mathrm{O}_{5}$, mientras que los tratamientos $\mathrm{MM}$ y $\mathrm{P} 2$ quedaron intermedios al no diferir de los de mayor y menor incidencia. En cuanto la severidad se observó una situación similar, los tratamientos de menor nivel los fueron Caldo Rizósfera y M6 y los de mayor nivel el testigo, $\mathrm{P} 1$ y $\mathrm{P}_{2} \mathrm{O}_{5}$, mientras que los tratamientos MM y P2 quedaron intermedios (Tabla 4).

Tabla 4. Incidencia y severidad de los síntomas de deficiencias de $\mathrm{P}$ en los diferentes tratamientos en el segundo muestreo el 15 de julio del 2017

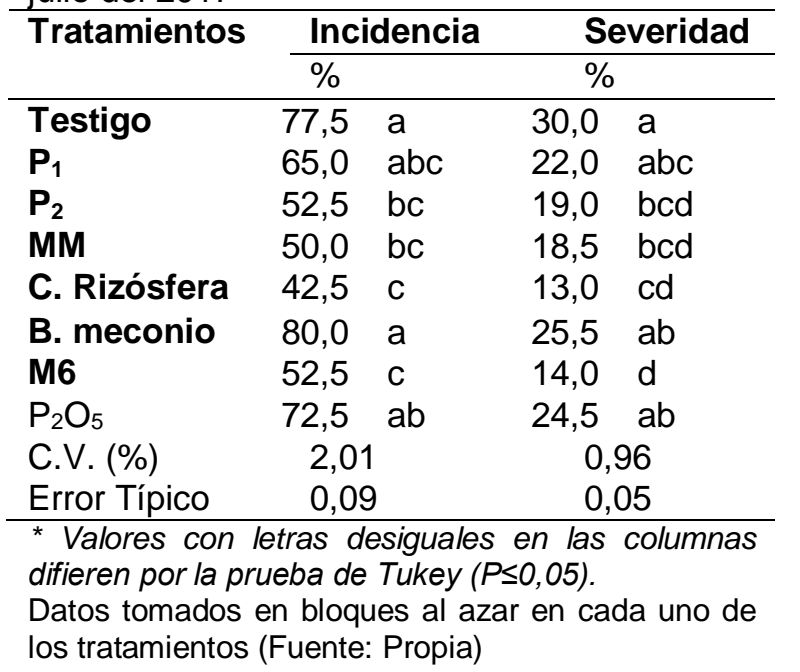

En el tercer muestreo en la semana 12 de haber iniciado las aplicaciones los tratamientos con menor incidencia resultaron Caldo Rizósfera y $\mathrm{M}_{6}$, aunque P2 y el testigo no se diferenciaron de estos y el de mayor incidencia fueron $\mathrm{P}_{2} \mathrm{O}_{5}$, B. meconio, $\mathrm{P} 1$ y $\mathrm{MM}$ que no se diferenciaron entre sí.

En cuanto al porcentaje de severidad Caldo rizosfera (10\%) y M6 (10\%) presentaron los menores niveles, aunque
P2 no se diferenció de estos, y los tratamientos de mayores niveles de severidad fueron $\mathrm{MM}$ y $\mathrm{P} 1$ y $\mathrm{P}_{2} \mathrm{O}_{5}$, aunque $\mathrm{P} 2$ y el testigo no se diferenciaron de estos (Tabla 5).

Tabla 5. Incidencia y severidad de los síntomas de deficiencias de $\mathrm{P}$ en los diferentes tratamientos en el tercer muestreo de la semana 12 (12 de agosto del 2017)

\begin{tabular}{lcccc}
\hline \multirow{2}{*}{ Tratamientos } & \multicolumn{2}{c}{ Incidencia } & \multicolumn{2}{c}{ severidad } \\
\cline { 2 - 5 } & \multicolumn{2}{c}{$\%$} & \multicolumn{2}{c}{$\%$} \\
\hline Testigo & 82,5 & bcd & 24,0 & ab \\
$\mathbf{P}_{1}$ & 77,5 & abc & 33,0 & $\mathrm{a}$ \\
$\mathbf{P}_{\mathbf{2}}$ & 80,0 & bcd & 27,5 & $\mathrm{abc}$ \\
MM & 90,0 & ab & 33,5 & $\mathrm{a}$ \\
C. Rizósfera & 35,0 & $\mathrm{~cd}$ & 10,0 & $\mathrm{c}$ \\
B. meconio & 95,0 & ab & 34,0 & $\mathrm{a}$ \\
M6 & 37,5 & $\mathrm{~d}$ & 11,0 & $\mathrm{bc}$ \\
$\mathbf{P}_{\mathbf{2}} \mathbf{O}_{5}$ & 100,0 & $\mathrm{a}$ & 38,5 & $\mathrm{a}$ \\
C.V. (\%) & \multicolumn{2}{c}{6,27} & & 2,48 \\
Error Típico & \multicolumn{2}{c}{0,18} & & 0,08 \\
\hline
\end{tabular}

* Valores con letras desiguales en las columnas difieren por la prueba de Tukey $(P \leq 0,05)$.

Datos tomados en bloques al azar en cada uno de los tratamientos (Fuente: Propia)

En el cuarto muestreo de la semana 16 la incidencia de los síntomas de carencia Fósforo fueron menores en los tratamientos Caldo Rizósfera y M6 que se diferenciaron del testigo y del resto de los tratamientos, mientras que los mayores niveles de incidencia lo mostraron el testigo, el biopreparado $\mathrm{P} 1$, el $\mathrm{B}$. de meconio, $\mathrm{MM}$ y el tratamiento de $\mathrm{P}_{2} \mathrm{O}_{5}$, aunque $\mathrm{P} 2$ se diferenció del testigo ni del tratamiento con $\mathrm{P}_{2} \mathrm{O}_{5}$. En cuanto la severidad se observó una situación similar, los tratamientos de menor nivel los fueron Caldo Rizósfera y M6 que se diferenciaron del resto de los tratamientos. Le siguieron en segundo orden $\mathrm{P}_{2} \mathrm{O}_{5}$ y $\mathrm{P} 2$ que se diferenciaron del resto de los tratamientos excepto de $\mathrm{MM}$, mientras que los que mayor severidad presentaron fueron el Testigo, MM y B. de meconio que no se diferenciaron entre sí (Tabla 6).

En el quinto muestreo de la semana 20 la incidencia de los síntomas de carencia 
fósforo fueron menores en los tratamientos Caldo rizosfera y $M 6$ que se diferenciaron del testigo y del resto de los tratamientos. Los mayores niveles de incidencia los presentaron el testigo y el tratamiento $\mathrm{P}_{2} \mathrm{O}_{5}$, mientras que los biopreparados $\mathrm{P} 1$, $\mathrm{P} 2$, MM y el $\mathrm{B}$. de meconio quedaron intermedios al diferir de los dos tratamientos de mayores valores de incidencia y de los menores. En cuanto la severidad se observó que los tratamientos de menor nivel los fueron Caldo Rizósfera y M6 que se diferenciaron del resto de los tratamientos. Le siguieron en segundo orden $\mathrm{P}_{2} \mathrm{O}_{5}$ y $\mathrm{P} 1$, aunque $\mathrm{P} 2$ no se diferenció de este último. Los tratamientos de mayor severidad fueron $B$. de meconio $y$ el Testigo que no se diferenciaron entre sí, aunque el B. de meconio no se diferenció de MM (Tabla 7).

Tabla 6. Incidencia y severidad de los síntomas de deficiencias de $\mathrm{P}$ en los diferentes tratamientos en el cuarto muestreo de la semana 16 (09 de agosto del 2017)

\begin{tabular}{lcccc} 
Tratamientos & \multicolumn{3}{c}{ Incidencia } & \multicolumn{2}{c}{ Severidad } \\
\cline { 2 - 5 } Testigo & \multicolumn{3}{c}{$\%$} & \multicolumn{2}{c}{$\%$} \\
$\mathbf{P}_{\mathbf{1}}$ & 95,0 & $\mathrm{a}$ & 46,0 & $\mathrm{a}$ \\
$\mathbf{P}_{\mathbf{2}}$ & 85,0 & $\mathrm{ab}$ & 30,5 & $\mathrm{bc}$ \\
MM & 77,5 & $\mathrm{~b}$ & 25,0 & $\mathrm{c}$ \\
C. Rizósfera & 87,5 & $\mathrm{ab}$ & 41,0 & $\mathrm{abc}$ \\
B. meconio & 40,0 & $\mathrm{c}$ & 12,0 & $\mathrm{~d}$ \\
M6 & 95,0 & $\mathrm{ab}$ & 39,0 & $\mathrm{ab}$ \\
$\mathbf{P}_{2} \mathrm{O}_{5}$ & 37,5 & $\mathrm{c}$ & 8,0 & $\mathrm{~d}$ \\
C.V. (\%) & 95,0 & $\mathrm{a}$ & 27,0 & $\mathrm{C}$ \\
Error Típico & 2,41 & & 1,63 & \\
& 0,12 & & 0,07 &
\end{tabular}

* Valores con letras desiguales en las columnas difieren por la prueba de Tukey $(P \leq 0,05)$.

Datos tomados en bloques al azar en cada uno de los tratamientos (Fuente: Propia)

Tabla 7. Incidencia y severidad de los síntomas de deficiencias de $\mathrm{P}$ en los diferentes tratamientos en el quinto muestreo de la semana 20 (07 de octubre del 2017)

\begin{tabular}{lcccc}
\hline Tratamientos & \multicolumn{2}{c}{ Incidencia } & \multicolumn{2}{c}{ Severidad } \\
\cline { 2 - 5 } & $\%$ & \multicolumn{3}{c}{$\%$} \\
\hline Testigo & 100,0 & $\mathrm{a}$ & 63,5 & $\mathrm{a}$ \\
$\mathbf{P}_{1}$ & 95,0 & $\mathrm{~b}$ & 37,0 & $\mathrm{de}$ \\
$\mathbf{P}_{2}$ & 92,5 & $\mathrm{~b}$ & 42,0 & $\mathrm{~cd}$
\end{tabular}

\begin{tabular}{lcccc} 
MM & 92,5 & b & 48,5 & bc \\
C. Rizósfera & 32,5 & $\mathrm{c}$ & 7,0 & $\mathrm{f}$ \\
B. meconio & 95,0 & $\mathrm{~b}$ & 55,5 & $\mathrm{ab}$ \\
M6 & 30,0 & $\mathrm{c}$ & 6,0 & $\mathrm{f}$ \\
$\mathrm{P}_{2} \mathrm{O}_{5}$ & 85,0 & $\mathrm{a}$ & 26,5 & $\mathrm{e}$ \\
\hline C.V. (\%) & 1,08 & & 0,99 & \\
Error Típico & 0,08 & & 0,05 & \\
\hline
\end{tabular}

* Valores con letras desiguales en las columnas difieren por la prueba de Tukey $(P \leq 0,05)$.

Datos tomados en bloques al azar en cada uno de los tratamientos (Fuente: Propia)

Los biopreparados Caldo Rizósfera y $\mathrm{M}_{6}$ demostraron poseer mejor potencial para sustituir la carencia de fósforo lo que se atribuye a que porque su producción se realiza a partir de microorganismos eficientes (Castellanos et al., 2018) que permiten solubilizar mejor los elementos para que la planta los absorba con mejor facilidad. Estos autores demostraron que los bioproductos Caldo Rizósfera y $\mathrm{M}_{6}$ contienen hongos, actinomicetos $y$ bacterias capaces de solubilizar el fósforo.

La dinámica de la variable incidencia en el tiempo durante el trascurso del experimento puso de manifiesto que las curvas de los biopreparados de Caldo Rizósfera y $M_{6}$ tuvieron unas tendencia a la disminución descendiendo desde más de $60 \%$ de incidencia a $30 \%$, mientras que los demás tratamientos las curvas se mantuvieron oscilando con una tendencia ligera al aumento de forma similar al testigo demostrando que no fueron eficaces para suplir la carencia de fósforo (Figura 1).

La dinámica de variable severidad de los síntomas en el tiempo, mostró una situación similar a la incidencia, ya que las curvas de los biopreparados de Caldo Rizósfera y $\mathrm{M}_{6}$ manifestaron una tendencia a la disminución reduciéndose desde valores mayores a $20 \%$ hasta valores entre 10 y $11 \%$, mientras que en los demás tratamientos las curvas se mantuvieron oscilando con una tendencia al aumento, siendo más pronunciada la pendiente en el testigo, ratificando que 
solo Caldo Rizósfera y $\mathrm{M}_{6}$ fueron eficaces para suplir la carencia de fósforo (Figura 2).

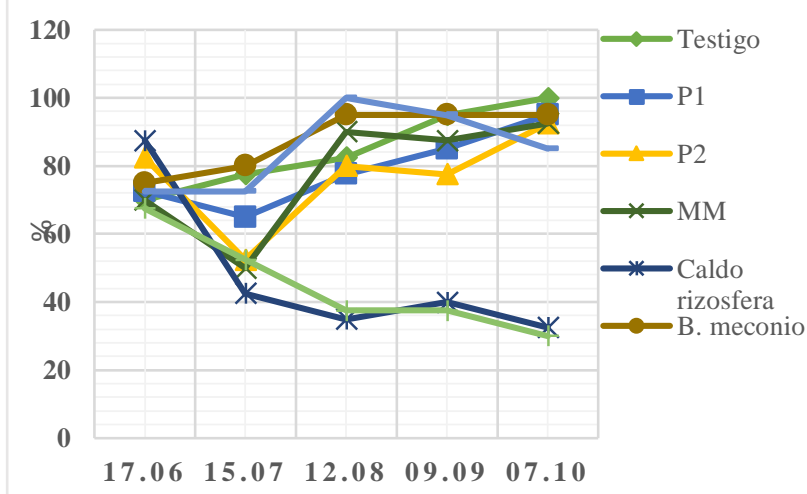

Figura 1. Dinámica de la Incidencia los síntomas de deficiencia de $\mathrm{P}$ en los tratamientos en los diferentes muestreos realizados. (Fuente: Propia)

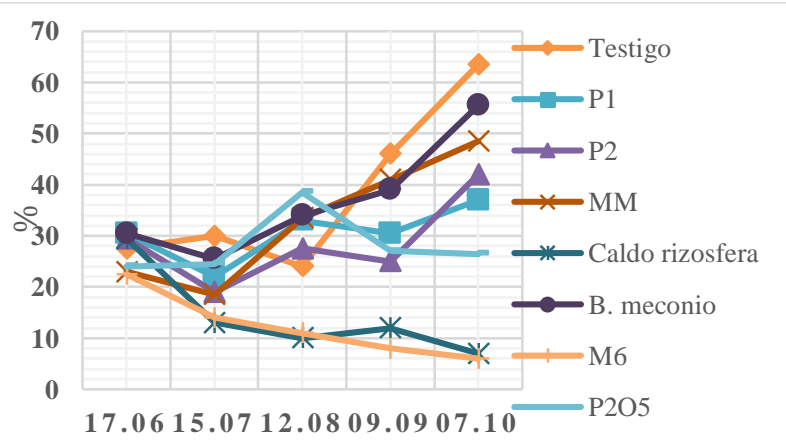

Figura 2. Dinámica de la severidad de los síntomas de deficiencia de $\mathrm{P}$ en los tratamientos en los diferentes muestreos realizados. (Fuente: propia)

La evaluación total durante cinco meses de aplicar los productos orgánicos se logró verificar que en cada recuento los biopreparados presentan mejores beneficios en cuanto a sustituir las necesidades de $P$ y los costos son más rentables comparados con el químico, también confrontados con el testigo es mejor hacer la aplicación de los productos orgánicos.

Los resultados con los dos bioproductos obtenidos localmente y la mejora de los suelos con la aplicación inicial de materia orgánica, permiten la disminución de fertilizantes fosforado de origen químico, permiten disminuir los residuos en la fruta de fresa y el imcato negativo en el medioambiente.

En el 2010 se realizó un trabajo comparando cultivo de fresa con manejo orgánico y otro con manejo químico ambos bajo invernadero, el cual obtuvo como resultado que el manejo orgánico donde se aplicaron los microrganismos eficientes estos permitían a largo plazo la asimilación de los elementos nutricionales, mientras que el tratamiento químico con el sistema de riego se producía un lavado del fosforo por escorrentía (Chiqui \& Lema, 2010).

Los biopreparados Caldo Rizósfera y $\mathrm{M}_{6}$ manifestaron los mayores valores de concentración de fosforo en el área foliar, demostrando diferencias con el testigo y con los demás tratamientos. Las menores concentraciones se obtuvieron para el testigo y los tratamientos con $\mathrm{P}_{1}$ y $\mathrm{P}_{2} \mathrm{O}_{5}$, mientras que los tratamientos $\mathrm{P}_{2}, \mathrm{MM}$ y $\mathrm{B}$. meconio presentaron un resultado intermedio de la concentración de fósforo (Tabla 8).

Tabla 8. Concentración de fósforo en el área foliar en cada tratamiento

\begin{tabular}{lcc}
\hline Tratamiento & $\begin{array}{l}\mathbf{m g} / \mathbf{k g} \text { de peso } \\
\text { de } \mathbf{P}_{\mathbf{2}} \mathbf{O}_{\mathbf{4}} \text { (ppm) }\end{array}$ \\
\hline Testigo & 9,5 & $\mathrm{C}$ \\
$\mathbf{P}_{\mathbf{1}}$ & 17,7 & $\mathrm{C}$ \\
$\mathbf{P}_{\mathbf{2}}$ & 21,2 & $\mathrm{bc}$ \\
$\mathbf{M M}$ & 22,1 & $\mathrm{bc}$ \\
C. Rizósfera & 35,3 & $\mathrm{a}$ \\
B. meconio & $21,3 \mathrm{bc}$ \\
M6 & $33,3 \mathrm{ab}$ \\
$\mathbf{P}_{\mathbf{2}} \mathbf{O}_{5}$ & 16,7 & $\mathrm{C}$ \\
C. de Variación (\%) & 20,01 \\
Error Típico & 2,68
\end{tabular}

Datos de la concentración de fósforo de la media para cada tratamiento tomada de los bloques al azar (Fuente: Propia) 
Estos resultados demuestran que bajo las condiciones de los suelos en estudio el $\mathrm{P}_{2} \mathrm{O}_{5}$ químico no resuelve la fertilización fosfórica, en primer lugar por lo bajo del $\mathrm{pH}$ que se fija al Aluminio y al hierro, en segundo lugar por la concentración baja de materia orgánica y el posible lavado de $\mathrm{P}_{2} \mathrm{O}_{5}$ por las Iluvias frecuentes durante la etapa del experimento, a diferencia de algunos de los otros tratamientos en que se favoreció la eficiencia de la absorción de $P$ del suelo cuando se aplicaron microrganismos solubilizadores de $P$, presentes en M6 y Caldo Rizósfera (Castellanos et al., 2017), así la materia orgánica aplicada, que permitían condiciones apropiadas para su desarrollo y función benéfica.

Se determinó un coeficiente de correlación significativa y negativa de 0,76 para la relación concentración de $P$ en el área foliar/incidencia, y significativa y negativa de 0,70 para la relación concentración de $P$ en el área foliar/severidad al final del experimento.

Se obtuvieron modelos de regresión lineales de la concentración de $P$ en función de la incidencia y severidad de los síntomas de deficiencias de $\mathrm{P}$ con coeficientes de determinación mayor de 0,5 (Tabla 9), o sea, que perimen explicar la variable dependiente de las independiente con un $50 \%$ o más de confiablidad, que si no es alto, da una medida de las relaciones que pudieran establecerse a futuro para que los agricultores puedan estimar los niveles de $P$ en la parte aérea del cultivo de fresa en función de la manifestación de los síntomas y tomar acciones para corregir la carencia.

En un trabajo comparando con cultivos de Fresa con manejo orgánico y otro con manejo químico ambos bajo invernadero fueron más costoso el manejo orgánico, pero con la producción se recompenso lo invertido (Chiqui \& Lema, 2010).

Tabla 9. Resultados de análisis de correlación y regresión entre concentración de fósforo y la incidencia y severidad de los síntomas de la deficiencia de $\mathrm{P}$ al final del experimento

\begin{tabular}{|c|c|c|c|c|}
\hline \multicolumn{2}{|c|}{ Variable } & \multirow[t]{2}{*}{$\mathbf{r}$} & \multirow{2}{*}{$\mathbf{R}^{2}$} & \multirow[t]{2}{*}{ Modelo } \\
\hline Depend. & Indepen. & & & \\
\hline \multirow{2}{*}{$\begin{array}{l}\text { Concentraci } \\
\text { ón } \\
\text { de fósforo } \\
\text { (C) }\end{array}$} & $\begin{array}{l}\text { Incidencia } \\
\text { (I) }\end{array}$ & $\begin{array}{l}- \\
0,7 \\
6\end{array}$ & $\begin{array}{l}0,5 \\
7\end{array}$ & $\begin{array}{l}\mathbf{C}=45,44- \\
10,04(\mathbf{I})\end{array}$ \\
\hline & $\begin{array}{l}\text { Severidad } \\
\text { (S) }\end{array}$ & $\begin{array}{l}- \\
0,7 \\
0\end{array}$ & $\begin{array}{l}0,5 \\
0\end{array}$ & $\begin{array}{l}\mathbf{C}=38,82- \\
13,66(\mathbf{S})\end{array}$ \\
\hline
\end{tabular}

r: coeficiente de regresión

$\mathrm{R}^{2}$ : Coeficiente de Determinación

Datos de las variables de la concentración de fósforo en cuanto a la correlación y determinación de cada independiente (Fuente: Propia)

Se realizó un trabajo de microrganismos benéficos como biofertilizantes en tomate, causando efectos positivos del estado nutricional de las plantas, aumentando en un $11 \%$ comparados con el testigo, obteniendo un gran nivel en las raíces de rizosfera (Alfonso, Leyva, \& Hernández, 2005).

En un diseño experimental comparando tres formas de fertilizar el cultivo de fresa bajo invernadero, una química, otra orgánico-mineral y la última orgánica. Presentando mejores resultados el experimento de orgánico-mineral en cuanto a crecimiento de corona, numero de estolones, frutos y hojas. Comparados con los demás ensayos (Romero et al, 2012; Vanegas et al, 2017) Rosatto, Castellanos, Caione, de Mello Reyes\& Silva (2014) recomendaron tambien el uso de biofertilizantes orgánicos y una fuente de materia orgánica para aumentar la solubilización del fósforo.

Sería recomendable validar en áreas más extensas del cultivo de la fresa el empleo de los bioproductos M6 y Caldo Rizósfera como alternativas orgánicas para suplir las deficiencias de fósforo en este cultivo de 
fresa acompañado con la aplicación de Materia Orgánica, así como continuar los estudios de los biopreparados que produce ASPAGRO en otros cultivos donde se presentes dificultades con la disponibilidad del fósforo con énfasis en M6 y Caldo Rizósfera, en aras de incorporar nuevas alternativas locales no químicas a la producción agrícola en el municipio de Pamplona.

\section{CONCLUSIONES}

De los seis productos orgánicos y el químico probados, se comprobó que Caldo Rizósfera y $M_{6}$ presentaron las mejores respuestas para disminuir la incidencia y severidad de la deficiencia de fósforo, comparados con el testigo y los demás tratamientos.

Los tratamientos Caldo Rizósfera y M6 se destacaron con relación a la mayor concentración de fósforo foliar, variable que demostró una correlación negativa y significativa con la incidencia y la severidad de los síntomas de la deficiencia de fósforo en los diferentes tratamientos al finalizar el experimento.

\section{Referencias Bibliográficas}

Alfonso, E., Leyva, A., \& Hernández, A. (2005). Microorganismos benéficos como biofertilizantes eficientes para el cultivo del tomate (Lycopersicon esculentum, Mill). Revista colombiana de Biotecnología, 47-54.

Angulo, W. J., Mendoza, J. A. y Uriel, H. U. (2017). Análisis de la vulnerabilidad por fenómenos de remoción en masa en la Cuenca Tanauca estudio de caso. Revista Ambiental Agua, Aire y Suelo. ISSN 1900-9178. Volumen (8), Numero

DOI: https://doi.org/10.24054/1900 9178.v2.n2.2017.3276
BID, B. I. (2009). Manual Práctico de Uso de EM. Convenio Fondo Especial de Japón. BID ATN/JO-10792 UR. Uruguay., 35.

Camargo, W. C. (2016). Modelación hidrologico-hidraulica de eventos de inundacion en el Rio Bogotá (sector tocanzipa-chia) usando HEC-RAS. Revista Ambiental Agua, Aire y Suelo. ISSN 19009178. Volumen (7), Numero (2). DOI: https://doi.org/10.24054/1900 9178.v2.n2.2016.3267

Cano, M. A. (2013). Estrategias biológicas para el manejo de enfermedades en el cultivo de fresa (Fragaria spp.). Revista Colombiana de Ciencias Hortícolas, 263-276.

Casierra, F. 2011. Crecimiento y eficiencia fotoquímica del fotosistema ii en plantas de fresa (fragaria sp.) afectadas por la calidad de la luz: implicaciones agronómicas. Revista U.D.C.A Actualidad \& Divulgación Científica, 43-53.

Castellanos, L., Céspedes, Sequeda, A., Mendoza, J.E., \& Niño, L.J. 2018. Caracterización microbiológica de cinco biopreparados artesanales. Revista Científica Agroecosistemas. , 6(3), 57-65.).

Castro, L., Murillo, M., Uribe, L., \& Mata, R. 2015. Inoculación al suelo con Pseudomonas fluorescens, Azospirillum oryzae, Bacillus subtilis $y$ Microorganismos de Montaña (MM) y su efecto sobre un sistema de rotación soya-tomate bajo condiciones de invernadero. Agornomia Costarriense, 21-36.

Chiqui, F., \& Lema, M. (2010). Evaluación del rendimiento en el cultivo de fresa (Fragaria sp) variedad oso grande, bajo invernadero mediante dos tipos de fertilización (orgánica y química) en la parroquia Octavio Cordero Palacios, Cantón Cuenca (Bachelor's thesis). Universidad politecnica saleciana, 68-79. 
Estrada, E., \& Iris, L. (2011). Respuestas bioquímicas en fresa al suministro de fósforo en forma de fosfito. Revista Chapingo. Serie horticultura.

Gualdron, C., \& Maldonado, B. (2017). Aproximación al caso de desarrollo local de la zona rural del municipio de Pamplona. FACE: Revista de la Facultad de Ciencias Económicas y Empresariales,, 142-156.

Gutierrez, T., Castellanos, C. y Hernández, N. (2016). El ordenamiento territorial frente a las consecuencias de los cambios climáticos. Revista Ambiental Agua, Aire y Suelo. ISSN 19009178. Volumen (7), Numero (2). DOI: https://doi.org/10.24054/1900 9178.v2.n2.2016.3338

Lara, C., \& Negrete, J. (2015). Efecto de un bioinoculante a partir de consorcios microbianos nativos fosfato solubilizadores, en el desarrollo de pastos Angleton (Dichantium aristatum). evista Colombiana de Biotecnología, 122130.

López, R. (2014). Las prácticas agrícolas de la Asociación Flores y Frutas de Huachi Grande y su incidencia en la calidad y productividad de fresas (fragaria vesca) variedad albión. (Master's thesis)., 150-185.

Luna, L. (2001). Producción, uso y manejo de bioestimulantes, abonos orgánicos, acondicionadores, y biofertilizantes a partir de fuentes no convencionales. CORPOICA, 58.

Mahecha, G.J., Castellanos, L. \& Céspedes, N. 2019. Incidencia y severidad de los síntomas de deficiencia de fósforo en el cultivo de fresa en las condiciones de Pamplona. 46, (1), 58-61.

Marquez, J.A. 2001. Organización y logística del mercado fresero onubense. Cuadernos Geográficos, 31, 115-128.
Medina, J., Pinzón, E., \& Cely, E. (2016). Efecto de sustratos orgánicos en plantas de fresa (Fragaria sp.) cv 'Albion'bajo condiciones de campo. Revista Ciencia y Agricultura, 1928.

Melo, J., Saavedra, S. y Ramón, J. A. (2017). Evaluación de la adsorcion de $\mathrm{CU}+2$ y azul de metileno en biosorbentes de bajo costo obtenidos a partir de biomasa residual de la agroindustria de cítricos. Revista Ambiental Agua, Aire y Suelo. ISSN 1900-9178. Volumen (8), Numero (2). DOI: https://doi.org/10.24054/1900 9178.v2.n2.2017.3277

Meneses, V. B., Álzate, D. y Mosquera, J. (2016). Sistema de optimización de las técnicas de planificación en agricultura de precisión por medio de drones. Revista Ambiental Agua, Aire y Suelo. ISSN 19009178. Volumen (7), Numero (2). DOI: https://doi.org/10.24054/1900 9178.v2.n2.2016.3268

Moreno, C. y Rueda, L. (2016). La educación ambiental como herramienta para la recuperación de la cobertura vegetal, mediante prácticas agro-ecológicas en la comunidad minera asograstorres, asociación de gravilleros de Sabana de Torres. Revista Ambiental Agua, Aire y Suelo. ISSN 1900-9178. Volumen (7), Numero DOI: https://doi.org/10.24054/1900 9178.v1.n1.2016.3260

Moreno, W. (2011). Aplicación de dos fosfitos artesanales en el cultivo de fresa (Fragaria vesca L.) Cevallos, ecuador. universidad técnica de ambato, 84-93.

Ortega, J. Y. (2016). Estructura ecológica principal de la cuenca del Río Pamplonita. Revista Ambiental Agua, Aire y Suelo. ISSN 19009178. Volumen (7), Numero (1). 
DOI: https://doi.org/10.24054/1900 9178.v1.n1.2016.3258

Rivera, H. U., Castellanos, C. y lbarra, A. (2017). Caracterización y cuantificación de los residuos sólidos realizado en el municipio de pamplona, $\mathrm{N}$ de $\mathrm{S}$, Colombia. Revista Ambiental Agua, Aire y Suelo. ISSN 1900-9178. Volumen (8), Numero

DOI: https://doi.org/10.24054/1900 9178.v2.n2.2017.3280

Romero, C. Ocampo-Mendoza, J., Sandoval-Castro, E., \& TobarReyes, J.R. 2012. Fertilización Orgánica-Mineral y Orgánica en el cultivo de fresa (Fragaria $x$ ananasa Duch.) bajo condiciones de invernadero. Ra Ximhai, 8, (3), 41-49.

Romero, C., \& et al. (2012). Fertilización Orgánica-Mineral y Orgánica en el cultivo de fresa (Fragaria $x$ ananasa Duch.) bajo condiciones de invernadero. Revista de Sociedad, Cultura y Desarrollo sustentable, 41-49.

Rosatto, L., Castellanos, L., Caione, G., de Mello, R., Reyes, A., \& Silva, N. (2014). Solubilización de fuentes de fósforo asociadas a un compuesto orgánico enriquecido con biofertilizantes. Agrociencia, 489-500.

Vanegas, D., Ramón, A. A. y Lizarazo, A. K. (2017). Comunidad y cultura ambiental. dinámicas de potenciación para un desarrollo sostenible y corresponsable. Revista Ambiental Agua, Aire y Suelo. ISSN 1900-9178. Volumen (8), Numero

DOI: https://doi.org/10.24054/1900 9178.v1.n1.2017.3271

Villamizar, C y Fernández, D. (2015). CARACTERIZACIÓN DE LOS PRODUCTORES DE DURAZNO (PRUNUS PÉRSICA (L) BATSCH) EN LAS PROVINCIAS DE PAMPLONA $Y$ RICAURTE,
NORTE DE SANTANDER. Revista Ambiental Agua, Aire y Suelo. Volumen (6), Numero (1). https://doi.org/10.24054/19009178. v1.n1.2015.3226 